\title{
HEMANGIOMA CAPILAR YUXTAPAPILAR Y RETINOPATÍA CENTRAL SEROSA: DESCRIPCIÓN DE UN CASO CON RESOLUCIÓN ESPONTÁNEA
}

\section{JUXTAPAPILLARY CAPILLARY HEMANGIOMA AND CENTRAL SEROUS RETINOPATHY: DESCRIPTION OF A CASE WITH SPONTANEOUS RESOLUTION}

\author{
PAREJA J ${ }^{1}$, GUZMÁN J ${ }^{2}$, CEDAZO M${ }^{1}$, VLEMING E ${ }^{1}$, JIMÉNEZ-PARRAS R ${ }^{1}$, TEUS MA ${ }^{3}$
}

\begin{abstract}
RESUMEN
Caso clínico: Paciente con pérdida de agudeza visual unilateral. Funduscópicamente se observó una masa compatible con hemangioma capilar yuxtapapilar (confirmada mediante angiofluoresceingrafía) y alteración del epitelio pigmentario y puntos de exudación en polo posterior compatibles con retinopatía central serosa bilateral.

En la evolución se produjo regresión espontánea tanto del hemangioma como de la retinopatía central serosa.

Discusión: La pérdida de visión en un paciente con hemangioma capilar yuxtapapilar se ha relacionado con complicaciones derivados del mismo, no habiendo sido descrita la asociación con retinopatía central serosa, como factor contribuyente.
\end{abstract}

Palabras clave: Hemangioma capilar yuxtapapilar, retinopatía central serosa, resolución espontánea, disminución de agudeza visual, complicaciones.

\begin{abstract}
Case report: A patient presented with a unilateral decrease of visual acuity and was found to have a juxtapapillary capillary hemangioma by ophthalmoscopy (confirmed by fluorescein angiography) and atrophy in the retinal pigmentary epithelium with leakage points suggesting bilateral central serous retinopathy. Spontaneous resolution of the hemangioma, as well as the central serous retinopathy, subsequently occurred.

Discussion: The decrease of visual acuity in a patient with a juxtapapillary capillary hemangioma has been associated with complications from the lesion itself, but an association of central serous retinopathy, as a possible predisposing factor, has not been previously described (Arch Soc Esp Oftalmol 2006; 81: 337-340).
\end{abstract}

Key words: Juxtapapillary capillary hemangioma, central serous retinopathy, spontaneous resolution, decrease in visual acuity, complications.

Recibido: 20/10/05. Aceptado: 12/6/06.

Servicio de Oftalmología. Hospital Universitario Príncipe de Asturias. Alcalá de Henares. Madrid. España.

1 Licenciado en Medicina.

2 Doctor en Medicina. Complejo Hospitalario Virgen de la Salud. Toledo. España.

3 Doctor en Medicina.

Comunicación presentada en el LXXXI Congreso de la S.E.O. (Zaragoza 2005).

Correspondencia:

Jesús Pareja Esteban

C/. Gurugú, portal 4, piso 2. ${ }^{\circ} \mathrm{A}$

28806 Alcalá de Henares (Madrid)

España

E-mail: txetupaes@hotmail.com 


\section{INTRODUCCIÓN}

Los hemangiomas capilares son hamartomas vasculares que pueden manifestarse de dos formas, bien como lesión periférica (tumor de von HippelLindau), o bien como tumor yuxtapapilar (1).

Pueden ser anomalías retinianas aisladas o pueden ser una manifestación mas del síndrome de von Hippel- Lindau (VHL).

Los hemangiomas yuxtapapilares presentan tres patrones posibles de crecimiento (2):

- Endofítico: crecen en la superficie del nervio óptico o de la retina, protruyendo en la cavidad vítrea.

- Exofítico: lesión nodular, anaranjada, crece en las capas externas de la retina.

- Sésil: apariencia aplanada, color gris-anaranjado. Depende de las capas medias retinianas.

Se describe un caso de hemangioma capilar yuxtapapilar de crecimiento endofítico, que debutó con disminución de agudeza visual.

\section{CASO CLÍNICO}

Varón de 51 años que acudió al servicio de urgencias por disminución de agudeza visual de su ojo izquierdo de cinco días de evolución.

Como antecedentes personales el paciente presentaba diabetes mellitus no insulino dependiente de seis años de evolución, así como haber padecido un traumatismo contuso en el mismo ojo 15 años antes.

Refería un cuadro de pérdida de visión asintomático de cinco días de evolución de su ojo izquierdo. La agudeza visual (AV) tomada mediante el test de Snellen era 1 para su ojo derecho y 0,5 para su ojo izquierdo, mejorando este último a 1 con una corrección esférica de $+1,5 \mathrm{D}$.

Tanto la motilidad ocular intrínseca como extrínseca, biomicroscopía anterior y tonometría del paciente eran normales.

La funduscopia del ojo a estudio presentaba una masa yuxtapapilar de color rojo-anaranjado brillante, sobreelevada, bien delimitada (fig. 1). También se apreció una alteración del epitelio pigmentario en región macular así como una alteración de la capa de fibras nerviosas superiores e inferiores. La exploración oftalmoscópica del ojo adelfo presentaba asimismo una leve alteración de epitelio pigmentario. Se realizó una angiografía fluoresceínica que demostró el relleno de un árbol vascular intratumoral compuesto de finos capilares con exudación en tiempos

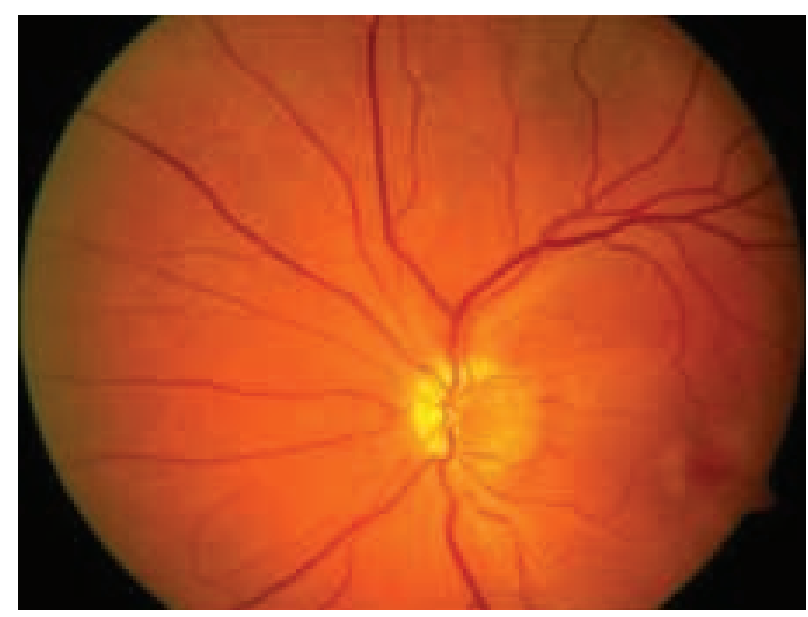

Fig. 1: Hemangioma capilar yuxtapapilar.

tardíos, confirmando la primera sospecha de hemangioma capilar yuxtapapilar. Se observaron puntos de fuga en polo posterior de ambos ojos, sugestivos de retinopatía central serosa (figs. 2 A-C).

Se realizó un campo visual SITA- Standard 24-2 (Zeiss-Humphrey Instruments, Dublin, CA) con visión corregida, estímulo III, hallándose un defecto infero-nasal del ojo izquierdo.

Cuatro semanas más tarde, la visión del ojo izquierdo era de 0,6 corregida ( +0.5 esf $+1 \times 48$ cil) 1; mientras que la visión del ojo derecho fue de 0,4 corregida (+0.5 esf $+0.25 \times 170$ cil) 0,6 . Una nueva angiografía fluoresceínica demostró que la disminución de agudeza visual del ojo derecho se debía a retinopatía central serosa.

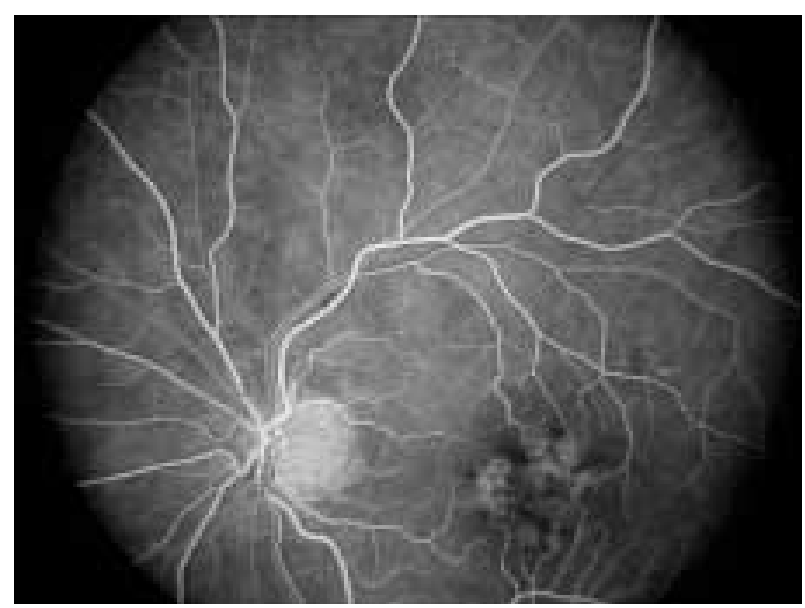

Fig. 2A: Tiempos precoces de AFG donde se aprecia el relleno capilar vascular del hemangioma así como lesiones cicatriciales del área macular del ojo izquierdo. 


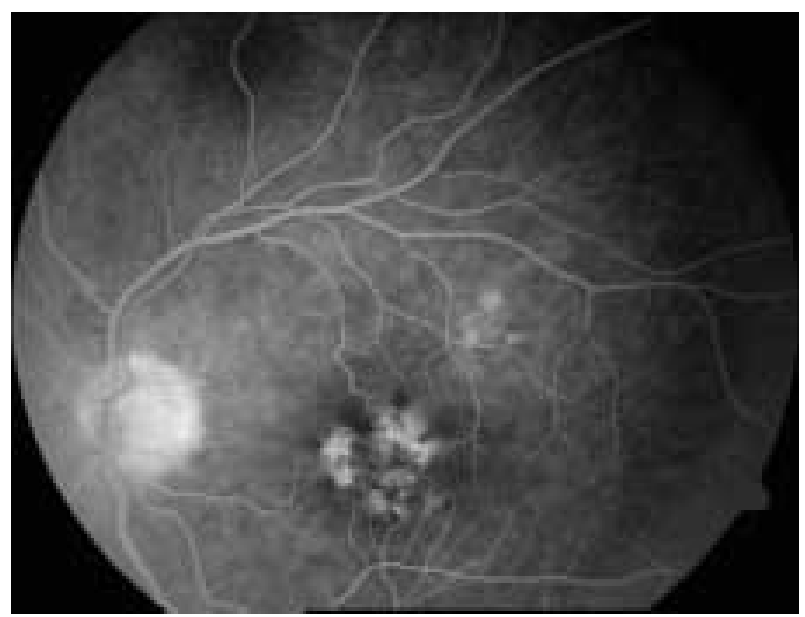

Fig. 2B: AFG en tiempos más avanzados: se aprecia fuga de la lesión tumoral yuxtapapilar así como puntos de fuga aislados en arcada vascular temporal superior.

Ocho semanas después del inicio del cuadro se encontró una regresión espontánea tanto del hemangioma capilar yuxtapapilar como de la retinopatía central serosa bilateral, regresando la AV a la 1 difícil en ambos ojos (fig. 3).

\section{DISCUSIÓN}

Los hemangiomas capilares son neoformaciones vasculares compuestos de numerosos vasos capilares, tapizados por un endotelio normal que se

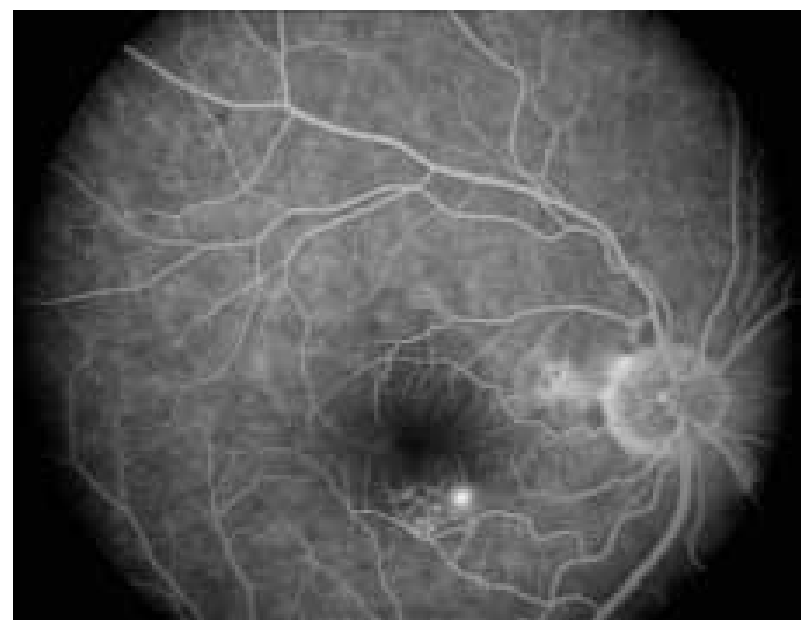

Fig. 2C: Imagen del ojo derecho donde apreciamos puntos de fuga en tiempos tardios en arcada vascular temporal inferior.

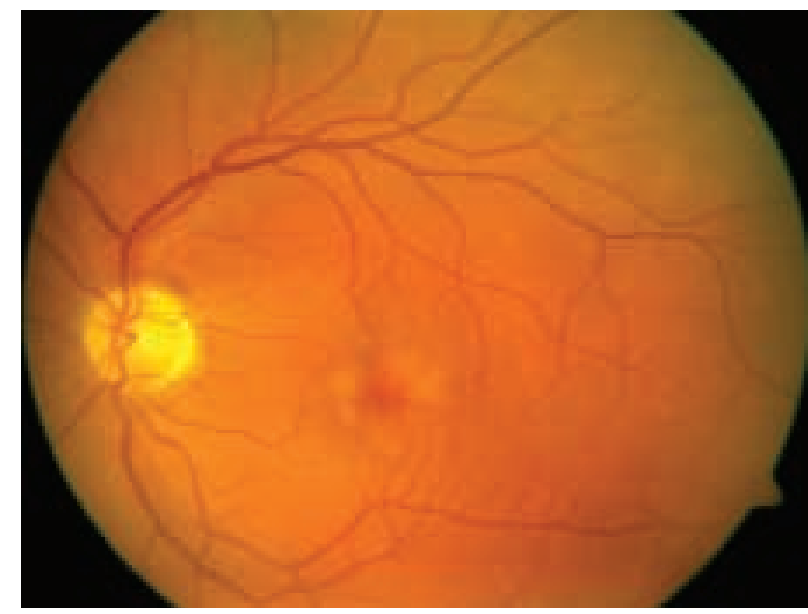

Fig. 3: Retinografía del ojo izquierdo donde se aprecia desaparición del hemangioma.

encuentra separado por células intersticiales poligonales vacuoladas de gran tamaño, conteniendo en su interior un material de aspecto lipídico. Su vascularización es dependiente tanto de la capa coroidea como de la retiniana.

En su evolución, los capilares constituyentes del hemangioma se vuelven incompetentes, dando lugar a una exudación macular progresiva sub e intrarretiniana que es en ocasiones motivo de su propio diagnóstico.

No es infrecuente la confusión del hemangioma capilar yuxtapapilar con papilitis, papiledema, neovascularización coroidea o coroiditis (2).

Los hemangiomas capilares yuxtapapilares, pueden presentarse de manera aislada o ser una manifestación más del síndrome de VHL (2). Por lo tanto es obligatorio ante todo paciente con hemangioma capilar, el realizar un estudio sistémico y multidisciplinar en busca de otras anomalías, así como screening familiar (3).

El hemangioma capilar yuxtapapilar puede permanecer asintomático o puede manifestarse como disminución de la agudeza visual (debida a desprendimiento retiniano seroso o traccional (3) formación de membrana epirretiniana o hemorragia).

Varios son los tratamientos que han sido propugnados para los hemangiomas capilares, tanto periféricos como yuxtapapilares.

En cuanto a los hemangiomas capilares de localización yuxtapapilar, son varios los autores que abogan por tomar una actitud no agresiva (4) dada la mayor tendencia a la estabilidad que presentan, 
habiendo sido descrita su desaparición espontánea. Hay que tener presente, sin embargo, que este tipo de lesión es de muy mal pronóstico, dada la dificultad en su diagnóstico temprano y la difícil accesibilidad al mismo.

En caso de progresión de la lesión o disminución en la agudeza visual, la fotocoagulación láser (argón o diodo) supone la terapia más empleada por los diferentes autores (5).

La retinopatía central serosa puede ser un factor contribuyente más a la disminución de la agudeza visual de pacientes con hemangioma capilar yuxtapapilar con regresión espontánea. Probablemente nos hallemos ante una asociación puramente debida al azar de dos patologías diferentes, si bien no podemos descartar un nexo común entre ambas aún no descrito.

\section{BIBLIOGRAFÍA}

1. McCabe CM, Flynn HW Jr, Shields CL, Shields JA, Regillo CD, McDonald RH, et al. Juxtapapillary capillary hemangiomas. Clinical features and visual acuity outcomes. Ophthalmology 2000; 107: 2240-2249.

2. Gass JD, Braunstein R. Sessile and exophytic capillary angiomas of the juxtapapillary retina and optic nerve head. Arch Ophthalmol 1980; 98: 1790-1797.

3. Malecha MA, Haik BG, Morris WR. Capillary hemangioma of the optic nerve head and juxtapapillary retina. Arch Ophthalmol 2000; 118: 289-291.

4. Singh AD, Nouri M, Shields CL, Shields JA, Perez N. Treatment of retinal capillary hemangioma. Ophthalmology 2002; 109: 1799-1806.

5. Schmidt-Erfurth UM, Kusserow C, Barbazetto IA, Laqua $H$. Benefits and complications of photodynamic therapy of papillary capillary hemangiomas. Ophthalmology 2002; 109: 1256-1266. 